\title{
Search for charged lepton flavor violation in J-PARC: The COMET experiment
}

Tianyu Xing ${ }^{* \dagger}$

Institute of High Energy Physics (IHEP), Beijing, People's Republic of China

E-mail: खingtydihep.ac.cn

COMET (COherent Muon to Electron Transition) is an experiment located in Japan, searching for flavour violation in the charged lepton sector, using the high intensity pulsed proton beam in J-PARC. COMET aims to search for the coherent neutrinoless process $\mu^{-}+N \longrightarrow e^{-}+N$. The single event sensitivity of the COMET experiment will be about $10^{-17}$, which is $10^{4}$ better than the current limit reported by the SINDRUM-II collaboration, $10^{-13}$ [四]. This article includes the physics motivation of COMET, a detailed description of the design and staged approach of the COMET experiment and an overview of the current status.

The 21st international workshop on neutrinos from accelerators (NuFact2019)

August 26 - August 31, 2019

Daegu, Korea

* Speaker.

${ }^{\dagger}$ On behalf of the COMET Collaboration 


\section{Introduction}

\subsection{Physics Motivation}

It is well understood that the Standard Model (SM) is a very successful model but with some mysteries yet unexplained, such as neutrino mass, dark matter and matter-antimatter asymmetry. All of these phenomena highlight the need for physics beyond the SM (BSM) and many BSM models, like SUSY, extra-dimension models and little Higgs models, predict sizable level of charged lepton flavour violation (CLFV). However, CLFV processes are highly prohibited in the SM with a branching ratio of $\mu \rightarrow e \gamma$ around $10^{-54}$ due to the tiny neutrino mass, which means the search is free from the SM background. Any evidence of CLFV signal would be a clear indication of new physics.

One of the most important CLFV process is the muon to electron conversion in the nuclear field. A "muonic atom" is formed when a muon stops in the target material. In the SM, nuclear muon capture and Decay in Orbit (DIO) are dominant. The mono-energetic signal electrons from $\mu-e$ conversions have an energy of $E_{\mu e}=m_{\mu}-B_{\mu}-E_{\text {recoil }}$, where $m_{\mu}$ is the mass of the muon, $B_{\mu}$ is the binding energy of the 1 s-state muonic atom and $E_{\text {recoil }}$ is the recoil energy of the nucleus. They can be identified by its energy, while the energy spectra of DIO is continuous. Considering this difference, the sensitivity could be improved by utilizing a high muon rate without suffering from any accidental background events and $\mu-e$ conversion is considered as a promising candidate to search for BSM physics.

\section{The COMET Experiment}

The COMET experiment searches for $\mu-e$ conversion in a muonic atom using a high-intensity pulsed proton beam from the J-PARC Main Ring (MR). The sensitivity goal is $2.6 \times 10^{-17}$, a $10^{4}$ improvement compared to the current limit[四].

\subsection{Experimental Design}

An $8 \mathrm{GeV} 56 \mathrm{~kW}$ proton beam from the J-PARC MR is extracted into a new proton beam line and directed to a pion production target. Pions produced in backward direction can be captured by a $5 T$ magnetic field. During the transportation through the $\mathrm{C}$ shape transport solenoid with $3 T$ magnetic field, the pions decay into muons, which are transported into stopping target section, and are stopped on 17 thin circular flat aluminum disks. Finally, the candidate signal electrons pass through a $\mathrm{C}$-shape electron spectrometer under a $1 T$ magnetic field and is detected by the straw tracker and the calorimeter. Three important design optimizations are adopted:

Firstly, to generate more muons, a thick graphite target with one or two hadron interaction lengths is chosen as the production target. To collect more pions and muons, an adiabatic capture magnet is used to collect backward particles. Finally the expected muon yield is $2 \times 10^{11} \mu / s$.

Secondly, two C-shape curved solenoid are chosen to get a cleaner muon beam. The curved solenoid will transport the charged particles along the vertical direction to the solenoid surface, so the beam background and signal can be separated in a better way than an $\mathrm{S}$ shaped transport solenoid. To adjust the muon beam into the center of solenoid, a dipole magnet is used. A collimator is put in the end of the transportation section for charge and momentum selection. 
Thirdly, the COMET experiment uses a straw tracker to provide a good momentum resolution. The detector system, including a straw tracker and a calorimeter, is located at the end of the electron beam line. The straw tracker is kept inside the vacuum and it consists of 5 stations as shown in Figure $\mathbb{W}$. Each station has 4 layers of $12 \mu \mathrm{m}$ thin straw tubes, which is light enough to provide the good momentum resolution. Lastly an electromagnetic calorimeter is located behind the straw tracker to provide trigger, TOF and PID informations.

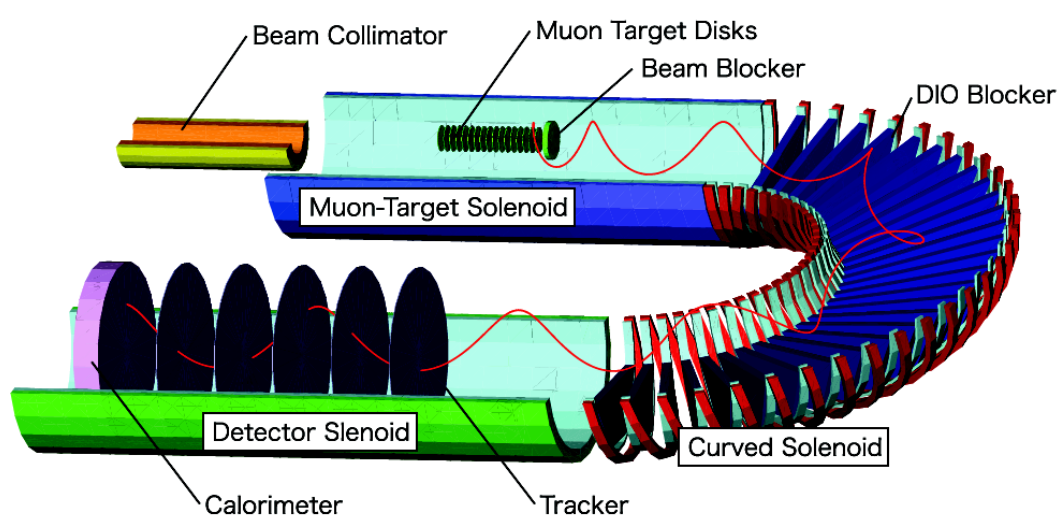

Figure 1: A schematic layout of the electron beam line and the detectors.

\subsection{Staged Approach of COMET}

The COMET experiment adopts a two stage approach to better control potential backgrounds and to better optimize the experiment, by precisely measuring muon beam. The difference between Phase-I and Phase-II is shown in Figure $\square$. Phase-I uses a $90^{\circ}$ bend pion transport solenoid and a specially designed cylindrical detector system that will be placed at the exit of this first bending, while the electron spectrometer is omitted. Also a straw tracker using $20 \mu \mathrm{m}$ thin straw tubes is developed for beam measurements. The Phase-I experiment is aiming to search for the $\mu-e$ conversion, but with a moderate single event sensitivity (S.E.S) of $3 \times 10^{-15}$.

A Cylindrical Detector system (CyDet) is specially designed for the $\mu-e$ conversion measurement for the Phase-I experiment. It consists of the Cylindrical Drift Chamber (CDC), the CyDet Trigger Hodoscope (CTH) and the stopping target. The CTH is composed of two layers of detectors, where the first layer is plastic scintillator for trigger and the second layer is Cerenkov counter for PID. The CDC is composed of 20 stereo layers of signal wire to provide track information. As the main tracker of COMET Phase-I, the CDC is required to have a momentum resolution of $400 \mathrm{keV}$. The stopping target in the center of the CDC is made of 17 aluminum disks with a thickness $200 \mu \mathrm{m}$ and a radius of $100 \mathrm{~mm}$.

\subsection{Background Rejection and Sensitivity}

In the COMET experiment, there are 3 categories of potential backgrounds in the signal region. The first category is the intrinsic physics background (mainly DIO), of which the spectrum peaks around $50 \mathrm{MeV}$ with a steep decrease towards higher energies extending to the end-point energy around $105 \mathrm{MeV}$. As the DIO end point energy is near the signal energy region, a good momentum resolution of less than $400 \mathrm{keV}$ is required to discriminate between the DIO and the signal. 


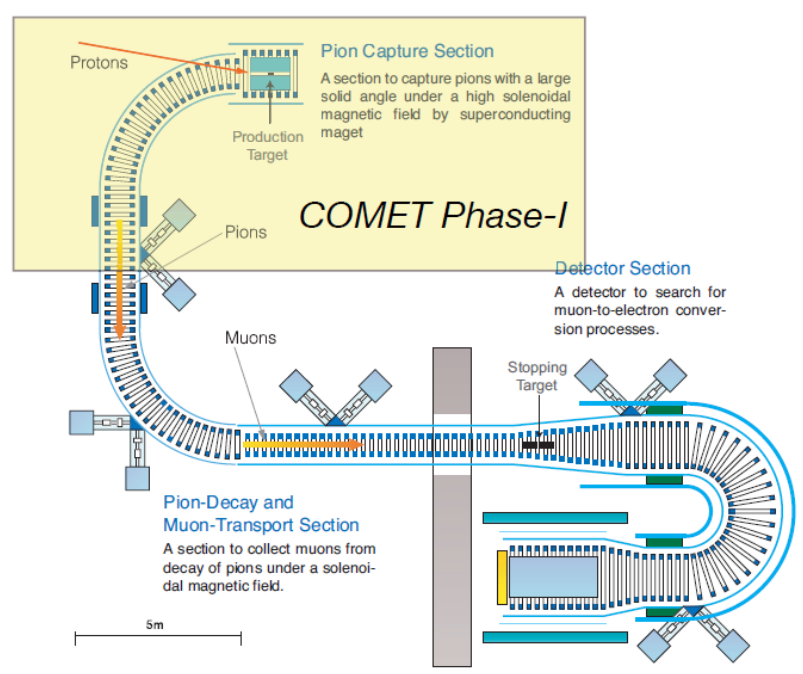

Figure 2: A schematic layout of COMET Phase-I and COMET Phase-II

The second source of background is the prompt beam related background. It can be suppressed by a pulsed beam and a delayed measurement window. As the $\mu-e$ conversion signals are delayed by the life time of the muonic atom, the delayed measurement window could reject most of the prompt background. The pulsed beam structure with $1.17 \mu \mathrm{s}$ between proton bunches of the COMET experiment is shown in Figure [3. Considering the life time of muon in the muonic atoms and muon stopping efficiency, aluminum is chosen as the target material. The life time of aluminum muonic atoms is $864 \mathrm{~ns}$, so a measurement time window from $700 \mathrm{~ns}$ to $1170 \mathrm{~ns}$ is set. Also the protons between pulses may break structure of beam, which requires the proton extinction must be controlled carefully.

The cosmic ray background can be suppressed by covering the stopping target and detector system with a veto counter. Also, in the Phase-I experiment, the cosmic rays can be fully reconstructed by the CDC, therefore, the effect of cosmic ray background can be clearly understood.

The performance of Phase-I experiment is estimated from a simulation study[ [ $]$. The overall acceptance of signal is $4.1 \%$, which means COMET Phase-I can reach a S.E.S $=3 \times 10^{-15}$ in about 150 days. The sum of accepted background is 0.032 , and the estimated average trigger rate is about $10 \mathrm{kHz}$. For COMET Phase-II, the experimental optimization is still going on. While the original sensitivity goal was $10^{-17}$ utilizing a $56 \mathrm{~kW}$ proton beam and one year of data acquisition, a recent study shows that a factor of 10 improvement will be feasible by full optimization of beam transportation and detector system acceptance while utilizing the same proton beam and beam time[3].

\section{Current Status}

COMET Phase-I is under construction while R\&D for COMET Phase-II is being carried out simultaneously:

The COMET experimental hall and installation yard are all completed, while cryogenic system is under construction. Proton beamline will be ready in the year of 2020. The pulsed structure 


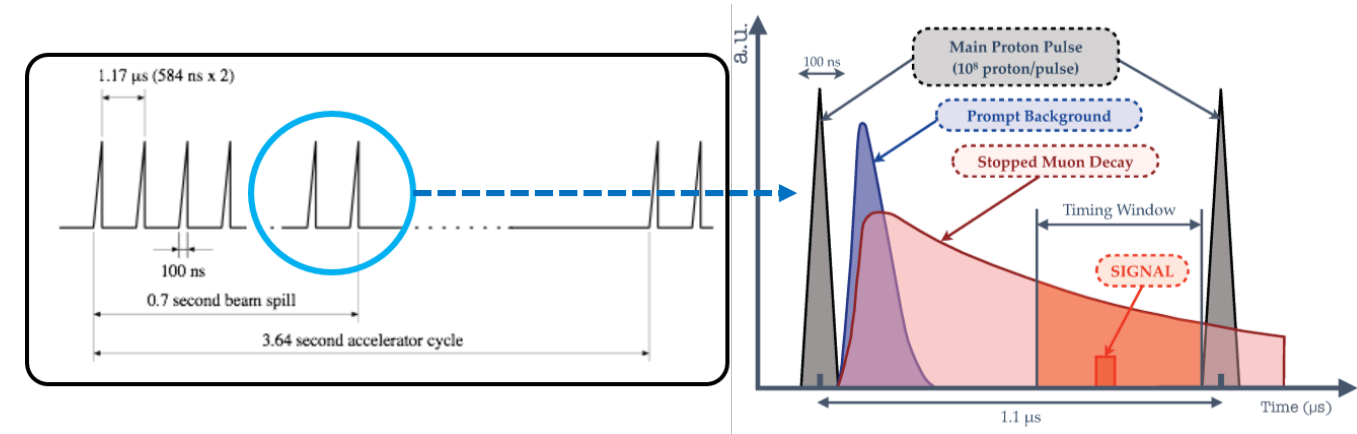

Figure 3: Left: Pulse structure of proton beam in J-PARC for COMET. Right: Time structure of beam.

with a low extinction factor of the proton beam at J-PARC is tested using the $8 \mathrm{GeV}$ proton beam. Results show that the extinction factor is better than $6 \times 10^{-11}$ which meets experimental requirements (the requirement of this extinction factor in COMET experiment is $O\left(10^{-9}\right)$ ). The last coil of capture solenoid is being bound. The transport solenoid has been installed and ready for cryogenic test. The detector solenoid coil and cryostat are ready and bridge solenoid has been delivered. A refrigerator test of cryostat system is completed and the helium transfer tube is under production.

On the other hand, the detector construction is also going on. The mass production for the straw tubes for phase-I is finished after the vacuum test for $20 \mu \mathrm{m}$ thin straw tubes. Furthermore, $12 \mu \mathrm{m}$ thin straw tubes for Phase-II are also produced and under testing. Two material have been tested as electromagnetic calorimeter modules and LYSO is chosen. The cosmic ray test result of CDC shows a spatial resolution of $150 \mu \mathrm{m}$ and a hit efficiency of $99 \%$. The main electronics for the COMET Phase-I have been produced and radiation test results are published or to be published. The electronics for the COMET Phase-II has finished design and radiation test results have been published.

\section{Summary}

COMET is an experiment located in J-PARC searching for $\mu-e$ conversion, which provides a powerful probe to BSM physics as a CLFV process. COMET plans to get a single event sensitivity of $2.6 \times 10^{-17}$, using a $56 \mathrm{~kW} 8 \mathrm{GeV}$ proton beam in 1 year beam time. In addition, a 10 times better sensitivity is possible with the ongoing optimizations. A staged plan is approached before achieving the final physics goals. The COMET Phase-I plans to directly measure the muon beam and search for $\mu-e$ conversion with single event sensitivity of $3 \times 10^{15}$, using a $3.2 \mathrm{~kW} 8 \mathrm{GeV}$ proton beam during 150 days of beam time. COMET Phase-I is under construction and Phase-II $\mathrm{R} \& \mathrm{D}$ study is going on.

\section{Acknowledge}

We thank KEK and J-PARC, Japan for their support of infrastructure and the operation of COMET. This work is supported in part by: Japan Society for the Promotion of Science (JSPS) KAKENHI Grant Nos. 25000004 and 18H05231; JSPS KAKENHI Grant No.JP17H06135; Belarusian Republican Foundation for Fundamental Research Grant F18R-006; National Natural 
Science Foundation of China (NSFC) under Contracts No. 11335009 and 11475208; Research program of the Institute of High Energy Physics (IHEP) under Contract No. Y3545111U2; the State Key Laboratory of Particle Detection and Electronics of IHEP, China, under 96 Contract No.H929420BTD; Supercomputer funding in Sun Yat-Sen University, China; National Institute of Nuclear Physics and Particle Physics (IN2P3), France; Shota Rustaveli National Science Foundation of Georgia (SRNSFG), grant No.DI-18-293; Deutsche Forschungsgemeinschaft grant STO 876/7-1 of Germany; Joint Institute for Nuclear Research (JINR), project COMET \#1134; Institute for Basic Science (IBS) of Republic of Korea under Project No. IBS-R017-D1-2018-a00; Ministry of Education and Science of the Russian Federation and by the Russian Fund for Basic Research grants: 17-02-01073, 18-52-00004; Science and Technology Facilities Council, United Kingdom; JSPS London Short Term Predoctoral Fellowship program, Daiwa AngloJapanese Foundation Small Grant; and Royal Society International Joint Projects Grant. Crucial computing support from all partners is gratefully acknowledged, in particular from CC-IN2P3, France; GridPP, United Kingdom; and Yandex Data Factory, Russia, which also contributed expertise on Machine Learning methods.

\section{References}

[1] W.H.Bertl et al. [SINDRUM-II Collaboration], Eur. Phys. J. C47(2006) p.337.

[2] The COMET Collaboration, Progress of Theoretical and Experimental Physics, v. 2020 (2020), p. 033C01 (arXiv:1812.09018).

[3] J.-C. Angélique et al., COMET - A submission to the 2020 update of the European Strategy for Particle on behalf of the COMET collaboration, arXiv:1812.07824v1. 